\title{
Diagnóstico de fallas estatóricas en motores de inducción de jaula de ardilla por medio de la corriente de secuencia negativa $^{1^{*}}$
}

\section{Stator Failure Diagnosis for Squirrel Cage Induction Motors through the Negative Sequence Current ${ }^{2}$}

\author{
Alejandro Paz Parra ${ }^{3}$ \\ José Luis Oslinger ${ }^{4}$ \\ Jairo Arcesio Palacios 5
}

DOI:10.11144/JaverIana.IYU18-1.DFEM

\begin{abstract}
' Fecha de recepción: 19 de noviembre de 2012. Fecha de aceptación: 13 de febrero de 2014. Este artículo se deriva de un proyecto de investigación denominado Diagnóstico de fallas en motores de inducción tipo jaula de ardilla mediante la aplicación de métodos híbridos, proyecto 1106-452-21072, desarrollado por el Grupo de Investigación en Conversión de Energía (Convergía), Universidad del Valle, Cali, Colombia.

* Los autores desean agradecer al Departamento Administrativo de Ciencia, Tecnología e Innovación de Colombia (Colciencias), por el apoyo financiero que permitió dotar a la Universidad del Valle del banco de ensayos para motores de inducción, del cual se pudieron obtener los resultados presentados en el presente artículo.

Adicionalmente, desean expresar especial agradecimiento a la recientemente fallecida ingeniera Martha Cecilia Amaya Enciso, PhD, por la contribución al trabajo hecha como profesora de la Escuela de Ingeniería Eléctrica y Electrónica de la Universidad del Valle, ya que fue promotora del laboratorio de diagnóstico de máquinas eléctricas de la Universidad del Valle, donde se realizaron los ensayos tratados en el presente trabajo. In memoriam.

${ }^{2}$ Reception date: November 19th, 2012. Acceptation date: February13th, 2014. This paper was obtained from a research project called Failure diagnosis in squirrel cage induction motors trough application of hybrid methods, number 1106452-21072, developed by the Energy Conversion Research Group (Convergia), from Universidad del Valle, Cali, Colombia. ${ }^{3}$ Ingeniero electricista, Universidad del Valle, Cali, Colombia. Magíster en Sistemas de Generación de energía, Universidad del Valle. Estudiante de doctorado en Ingeniería con énfasis en Ingeniería Eléctrica y Electrónica, Universidad del Valle. Profesor asistente, Pontificia Universidad Javeriana, Cali, Colombia. Correo electrónico: apaz@javerianacali.edu.co.

${ }^{4}$ Ingeniero electricista, Universidad del Valle, Cali, Colombia. Doctor en Ingeniería, Universidad del Valle. Profesor, Universidad del Valle. Investigador en el grupo Conversión de Energía (Convergía), Universidad del Valle.

Correo electrónico: jose.oslinger@correounivalle.edu.co.

${ }^{5}$ Ingeniero electricista, Universidad del Valle, Cali, Colombia. Doctor en Sistemas de Generación de Energía, Universidad Politécnica de Madrid, España. Profesor, Universidad del Valle. Investigador en el grupo Conversión de Energía (Convergía), Universidad del Valle. Correo electrónico: jairo.palacios@correounivalle.edu.co.
\end{abstract}




\section{啝以门料}

En este trabajo se presenta un modelo matemático para obtener un indicador de falla en el estator basado en la corriente de secuencia negativa, el cual puede utilizarse en sistemas de diagnóstico en línea al separar los efectos del desbalance de voltaje y las asimetrías constructivas de la máquina. Se presentan resultados sobre la aplicación experimental de la técnica propuesta mediante una alternativa computacionalmente económica. Los resultados de la aplicación del modelo se validan mediante ensayos de laboratorio en un motor modificado especialmente y se obtienen resultados aplicados a dos motores de mayor potencia, los cuales fueron exitosamente diagnosticados por fallas en el estator. El resultado del trabajo es un modelo de gran aplicabilidad por ser poco invasivo y de bajo costo computacional, para diagnosticar un tipo de falla de evolución rápida que lleva en un corto tiempo al colapso térmico del bobinado de la máquina con los consecuentes costos de reparación.

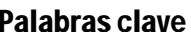

Motor de inducción, diagnóstico, falla estatórica, factor de desequilibrio de tensiones, corto circuito.

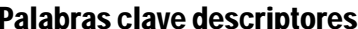

Motores eléctricos de inducción, diagnóstico de fallas, tensiones, estatores, cortocircuitos.

\section{>}

This paper presents a mathematical model to obtain a failure indicator of stator faults based on the negative sequence component of the electric current. The model can be used in online diagnosis systems to separate the effects of voltage unbalance factor and the constructive asymmetries of the machine. Results of the application of the model were validated trough laboratory tests made into a specially modified motor and results were applied to success diagnosis of two motors of higher electrical power. As a main result of the work, a low computational model was obtained to diagnose a quick evolution failure that can evolve quickly to a thermal collapse of the stator winding with consequent repair costs.

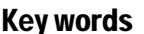

Induction motor, diagnosis, Inter-turn stator fault, unbalanced voltage factor, Short circuit.

\section{䗇 D}

Electric motors, fault diagnosis, stresses, stators, short circuits. 


\section{Introducción}

Los métodos de detección de fallas en el estator se basan en los signos expuestos por dicha condición, algunos de los cuales se evidencian mediante inspección visual directa (chispas, descargas parciales, deterioro del aislamiento, descamado, contaminación) (IEEE Instrumentation and Measurement Society, 2004). Los métodos de inspección visual carecen de confiabilidad en la detección en estado incipiente y no permiten detectar un cortocircuito entre espiras de una misma bobina. Para cuando los síntomas se hacen notables, es porque normalmente la falla ha evolucionado de forma rápida y pone en peligro la vida del motor y toda la producción asociada (Bouzid y Champenois, 2012).

En reemplazo de la inspección directa, se han desarrollado métodos como la inspección eléctrica de los aislamientos a través de la prueba de resistencia de aislamiento, la medida del índice de polarización, la medida de descargas parciales o la prueba de alto voltaje; pero según recomienda el estándar IEEE 43-2000 (Society, Electric Machinery Committee of the IEEE Power Engineering, 2000), la aplicación periódica de estas pruebas no es recomendable, porque los altos esfuerzos electromagnéticos a los que se somete el material aislante pueden deteriorarlo gravemente.

Existen alternativas de diagnóstico en línea para fallas ubicadas en el estator de la máquina de inducción centradas en las corrientes de alimentación, entre las que se encuentran el análisis en el dominio de la frecuencia en el espectro de Fourier, tanto real como complejo, y las transformadas wavelet; pero estas requieren un esfuerzo computacional importante para el cual no siempre existen recursos disponibles (Nandi, Toliyat y Xiaodong, 2005).

Cuando ocurren fallas en el devanado del estator, casi siempre se encuentran acompañadas por un desbalance en las corrientes de alimentación, el cual se manifiesta a través de la corriente de secuencia negativa; las modificaciones en magnitud y fase de esta corriente se usan ampliamente como base para el diagnóstico, pero a lo largo de los años se ha demostrado que esta corriente no es un indicador totalmente fiable, ya que existen muchas fuentes de variabilidad, 
como los desbalances de voltaje y las asimetrías constructivas de la máquina (Bouzid y Champenois, 2012; Bakhri et al., 2010).

Dentro de las fallas ocurridas en el estator de un motor de inducción, ocupa un papel preponderante la falla por cortocircuito entre espiras, ya que el aislamiento entre dos espiras de una misma bobina suele ser débil, y bajo ciertas condiciones de operación, puede estar sometido a grandes esfuerzos electromagnéticos; esto lo convierte en el eslabón más débil del sistema total de aislamiento de un motor. Las consecuencias de un cortocircuito entre espiras pueden ser catastróficas, porque aparece una corriente circulante localizada de un valor significativamente alto (varias veces la corriente nominal), que no se refleja en los terminales del motor y que produce un efecto de aumento local de la temperatura. El incremento de temperatura hace que el aislamiento exterior se deteriore progresivamente, lo que lleva en poco tiempo a un cortocircuito de mayor envergadura, como el que ocurre entre fase y tierra o entre bobinas de diferentes fases dentro de la misma ranura (Bouzid y Champenois, 2012). El signo exterior de la falla es que el motor se quema, pero el factor determinante de la falla catastrófica es la pérdida de aislamiento entre espiras.

La técnica aplicada en el presente trabajo fue propuesta originalmente por Bakhri et al. (2010) y validada por Bouzid y Champenois (2012), por lo que se hace una apropiación tecnológica de esta y una validación de los resultados obtenidos por los autores anteriores con base en los recursos disponibles. El principal aporte del trabajo se encuentra en la depuración del modelo y su aplicación para demostrar su operatividad con bajos costos computacionales.

\section{Marco referencial}

En el marco de referencia de componentes simétricas negativa positiva y cero (marco NP0), tradicionalmente, se usa como indicador la corriente de secuencia negativa, por ser la expresión más directa de la presencia de asimetrías en el devanado generadas por fallas de cortocircuito entre espiras (Villada, Cadavid y Velilla, 2010; Veruchi y Acosta, 2007).

Las componentes de secuencia en el marco referencial NP0 se encuentran determinadas por la transformación expresada en la ecuación:

$$
\left[\begin{array}{c}
V_{0} \\
V_{p} \\
V_{n}
\end{array}\right]=\frac{1}{3}\left[\begin{array}{ccc}
1 & 1 & 1 \\
1 & a & a^{2} \\
1 & a^{2} & a
\end{array}\right]\left[\begin{array}{c}
V_{a} \\
V_{b} \\
V_{c}
\end{array}\right]
$$


$\left[\begin{array}{l}I_{0} \\ I_{p} \\ I_{n}\end{array}\right]=\frac{1}{3}\left[\begin{array}{ccc}1 & 1 & 1 \\ 1 & a & a^{2} \\ 1 & a^{2} & a\end{array}\right]\left[\begin{array}{c}I_{a} \\ I_{b} \\ I_{c}\end{array}\right]$

Donde: $a=e^{j \frac{2 \pi}{3}}$

Los valores $V_{a}, V_{b}, V_{c}, I_{a}, I_{b}$ e $I_{c}$ corresponden a los valores fasoriales de voltaje y corriente y los valores $V_{p}, V_{n}, V_{0}, I_{p}, I_{n}$ e $I_{0}$ son las componentes de secuencia positiva, negativa y cero, de los cuales se extrae la corriente $I_{n}$ como indicador de falla.

En caso de no disponer de los voltajes de fase, la transformación se puede hacer con voltajes de línea, pero las corrientes que se van a usar deben ser las corrientes de fase, y la metodología es aplicable a motores conectados en $Y$.

En condiciones de perfecta simetría, el voltaje y la corriente de secuencia negativa deberían ser nulos, pero en una operación real, incluso cuando los voltajes de alimentación se encuentran balanceados, aparece una corriente de secuencia negativa que obedece a las asimetrías naturales del proceso de bobinado del motor (impedancias ligeramente desbalanceadas por fase).

Por otra parte, si la fuente de alimentación no presenta voltajes balanceados, aparece una componente de voltaje negativa que induce una corriente desbalanceada y genera una componente de corriente negativa que circula por los devanados. La corriente total de secuencia negativa que se obtiene a partir de las corrientes de fase tiene identificadas tres componentes:

$$
I_{n}=I_{s f}+I_{0 n}+I_{V U F}
$$

\section{Donde:}

$I_{s f} \quad$ componente de la corriente generada por la condición de falla.

$I_{0 n}:$ corriente de secuencia negativa debida a imperfecciones, defectos de fabricación o bobinado inherentes al motor, es decir, no relacionada con la situación de falla o de alimentación asimétrica.

$I_{\mathrm{vUF}}$ : corriente de secuencia negativa asociada al desequilibrio de tensiones de alimentación expresado a través del coeficiente VUF como indicador de dicho desbalance, según lo define el STD IEEE 1159-2009 (IEEE Power \& Energy Society, 2009). 


$$
\% V U F=\frac{U_{n}}{U_{p}} \%
$$

En la figura 1 se muestra el circuito equivalente del motor para secuencia positiva y negativa. Las componentes de resistencia y reactancia del rotor se refieren al estator usando el deslizamiento y se desprecian las pérdidas en el núcleo al reemplazar la rama de magnetización por una reactancia.

Figura 1. Circuito equivalente de secuencia: a) positiva y b) negativa

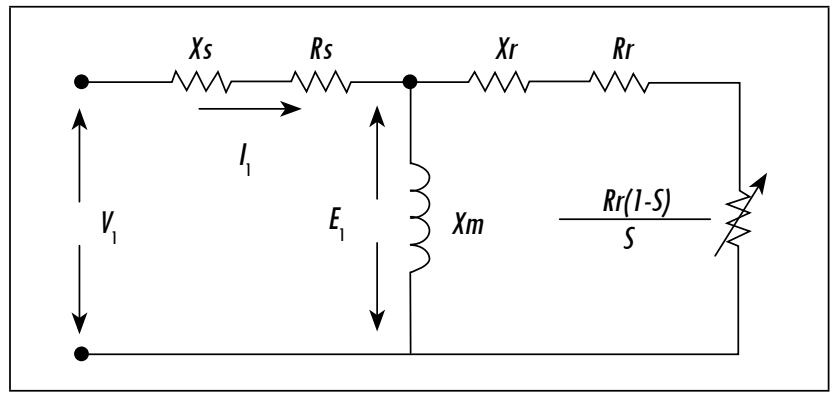

a)

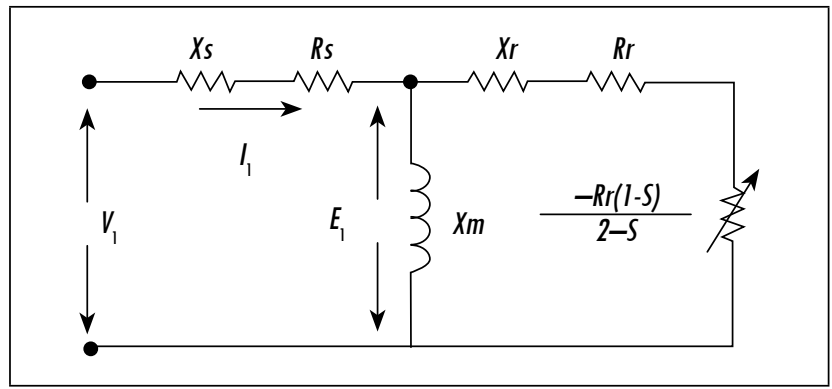

b)

Fuente: presentación propia de los autores.

La componente variable de la resistencia del rotor reflejada representa la potencia mecánica del motor en la secuencia positiva y negativa respectivamente $\left(R_{p}\right.$ y $\left.R_{n}\right)$. La resistencia $R_{r}$ es la resistencia de rotor bloqueado obtenido en la prueba del mismo nombre según aparece en el IEEE STD 112-2004 (IEEE Power Engineering Society, 2004).

Un análisis de dicha componente en relación con la resistencia $R_{r}$ muestra que la resistencia de secuencia negativa $\left(R_{n}\right)$ es menor en magnitud, a pesar de 
que la resistencia de secuencia positiva $\left(R_{p}\right)$ es más sensible frente a cambios de deslizamiento, como se muestra en la tabla 1 .

Tabla l. Componente variable de la resistencia de rotor en función del deslizamiento para secuencia positiva $\left(R_{p}\right)$ y negativa $\left(R_{n}\right)$

\begin{tabular}{|c|c|c|c|}
\hline$S(\%)$ & $R_{p} / R_{r}$ & $R_{n} / R_{r}$ & $R_{p} / R_{n}$ \\
\hline 1 & 99 & 0,50 & 200 \\
\hline 2 & 49 & 0,49 & 100 \\
\hline 3 & 32 & 0,49 & 65 \\
\hline 4 & 24 & 0,49 & 49 \\
\hline 5 & 19 & 0,49 & 39 \\
\hline 6 & 16 & 0,48 & 33 \\
\hline 7 & 13 & 0,48 & 27 \\
\hline 8 & 12 & 0,48 & 25 \\
\hline 9 & 10 & 0,48 & 21 \\
\hline 10 & 9 & 0,47 & 20 \\
\hline
\end{tabular}

Fuente: presentación propia de los autores.

Como se puede apreciar en la tabla 1, la relación entre la resistencia de secuencia positiva y negativa es alta, lo cual indica que, a pesar de que se tenga un voltaje de secuencia negativa bajo, se genera una corriente de secuencia negativa alta en la zona de bajo deslizamiento. Esta dependencia, sin embargo, no es tan directa, ya que en el circuito de secuencia negativa se aprecia una reactancia propia del devanado del rotor, proporcional al doble de la frecuencia de alimentación. Esta reactancia limita la corriente de secuencia negativa por el rotor y en su consecuencia su valor reflejado al estator. De esta forma, un incremento en el factor de desbalance de voltajes se refleja como un incremento proporcional en la corriente de secuencia negativa. La constante de proporcionalidad depende del tipo de diseño del motor y de sus parámetros eléctricos (Bakhri et al., 2010).

\section{Metodología}

Se usó un motor trifásico de 3 caballos de potencia, 220 V, 8.4 A, 1740 rpm, especialmente modificado para introducir fallas por cortocircuito entre espiras con el fin de verificar la relación entre el nivel de cortocircuito y la corriente de secuencia negativa. El motor tiene 2 grupos de bobinas por fase, las cuales permiten hacer conexiones en Y, YY, D y DD para voltajes de 440 V, 220 V, 380 V y 127 V, respectivamente. Durante los ensayos se usó la configuración YY. 
En el proceso de bobinado del motor se introdujeron terminales intermedios en el devanado como se muestra en la figura 2. Estos terminales permiten hacer cortocircuitos entre espiras diferentes de una misma fase en cantidades de 2, 5 , 10 y 14 espiras; así como medir la corriente de falla y las corrientes y voltajes de fase en diferentes condiciones de carga o vacío. El bobinado modificado se muestra en la figura 3.

Figura 2. Bobinado conectado en YY con TAPS para introducir corto entre espiras

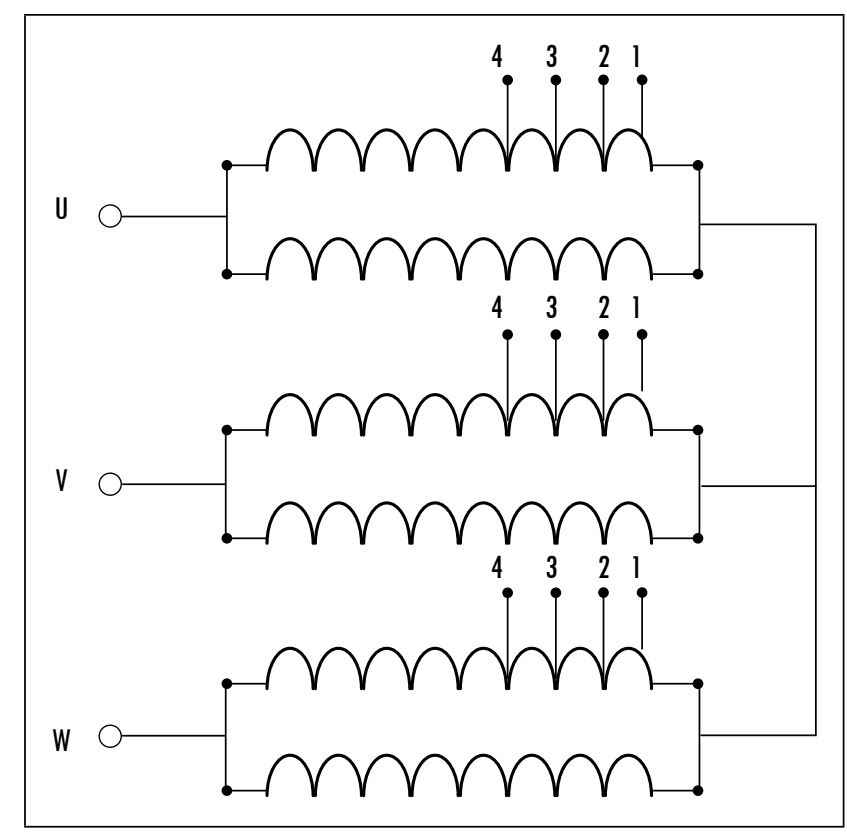

Fuente: presentación propia de los autores.

En el nuevo tablero de conexiones se encuentran a mano izquierda los conectores usuales de la bornera que dan acceso a la modificación de la conexión entre los grupos de bobinas para cambiar el nivel de tensión y el modo de operación; mientras que a mano derecha se agregaron unos puntos conectados a las derivaciones hechas para generar los cortocircuitos entre las espiras, según se aprecia en la figura 4. 
Figura 3. Vista lateral del bobinado del motor con los terminales insertados para hacer los corto-circuitos

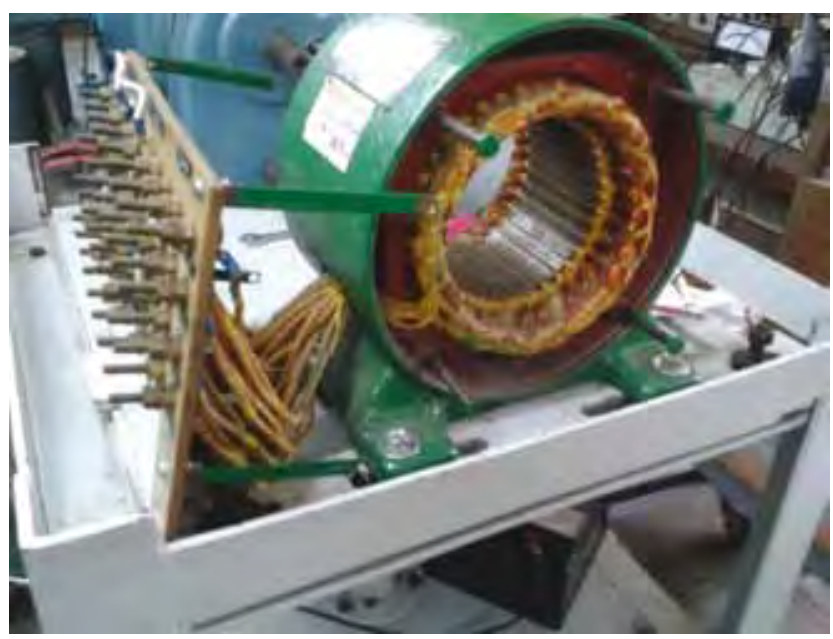

Fuente: presentación propia de los autores.

Figura 4. Tablero de conexiones del motor modificado para introducir las diferentes condiciones de falla

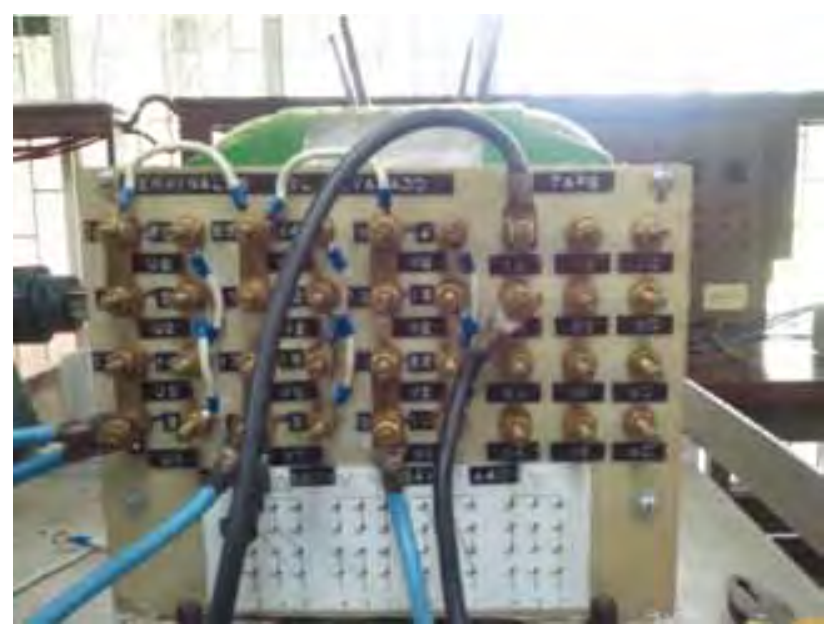

Fuente: presentación propia de los autores.

Se usó una resistencia limitadora de la corriente de falla para prevenir daños permanentes en el motor durante el proceso de introducción de la falla, como se muestra en la figura 5 . 
Figura 5. Esquema del montaje experimental para realizar las mediciones

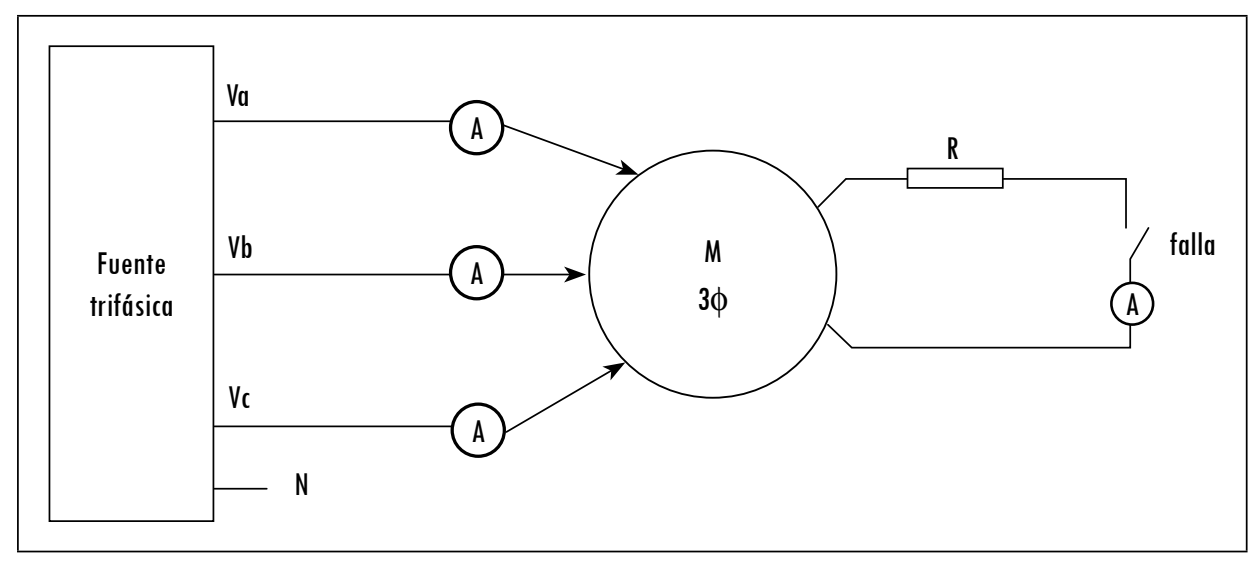

Fuente: presentación propia de los autores.

En un trabajo previamente publicado se analizó el método de los elementos finitos, del cual se extrajo información importante que ayudó a guiar el proceso de experimentación y a validar el modelo obtenido (Díaz et al., 2010), pero en esa publicación se usó como referencia el valor de la impedancia de secuencia negativa según lo propusieron Veruchi y Acosta (2007); mientras en este trabajo se hace hincapié en la corriente de secuencia negativa como los proponen Bakhri et al. (2010) y Bouzid y Champenois (2012). Los resultados de esta investigación fueron presentados previamente en una conferencia internacional, pero en el presente documento se amplía la discusión de resultados y sus detalles operativos (Paz Parra et al., 2012).

Para aislar las tres componentes identificados en la corriente de secuencia negativa se realizaron los siguientes experimentos:

- Se midió la corriente de secuencia negativa debido a asimetrías inherentes al motor: se hicieron ensayos de falla en vacío en el motor con 2, 5, 10 y 14 espiras en corto. En todos los casos se usó un factor de desequilibrio de tensiones bajo $(<1 \%)$ de acuerdo con el STD 112-2004 IEEE (IEEE Power Engineering Society, 2004), se midió la corriente de secuencia negativa y se identificó la tendencia cuando el número de espiras en falla tiende a cero en los diferentes ensayos.

- Se midió la corriente de secuencia negativa asociada al desequilibrio de tensiones: se introdujeron desbalances de voltaje inferiores al 4,2\%, sin presencia de fallas en la máquina. Se descontó la corriente generada por asimetrías propias del motor (obtenida en el paso anterior), con lo cual se obtuvo la componente generada exclusivamente por el desequilibrio 
de tensiones. Se planteó una función que relaciona esta componente de corriente con el VUF expresado en porcentaje.

- Finalmente, se introdujeron condiciones de falla con diferentes factores de desequilibrio de tensiones y se midió la corriente total de secuencia negativa, de la cual se extraen las componentes obtenidas en los pasos anteriores para tener una corriente que verdaderamente refleja la condición de falla.

Una vez realizado este conjunto de experimento se determinó un factor de severidad asociado a cada nivel de falla introducido y representado en la magnitud de la corriente $I_{s f}$ la cual se puede expresar en amperios o en valores por unidad.

Finalmente, con los resultados obtenidos se midieron varios motores que se encontraban trabajando en campo y se identificó que dos de ellos mostraban claros indicios de falla en estator por el método propuesto. Los resultados de esos dos motores se muestran en este artículo.

\section{Resultados}

Para el análisis de componentes de secuencia se usó una aplicación desarrollada por los autores en modo gráfico de MATLAB ${ }^{\circledR}$. La interfaz desarrollada con los resultados obtenidos para el motor con 14 espiras en cortocircuito se muestra en la figura 5 . Se puede apreciar que el valor de la corriente de secuencia negativa es aproximadamente el $10 \%$ de la corriente nominal.

Figura 6. Interfaz gráfica usada para analizar componentes de secuencia

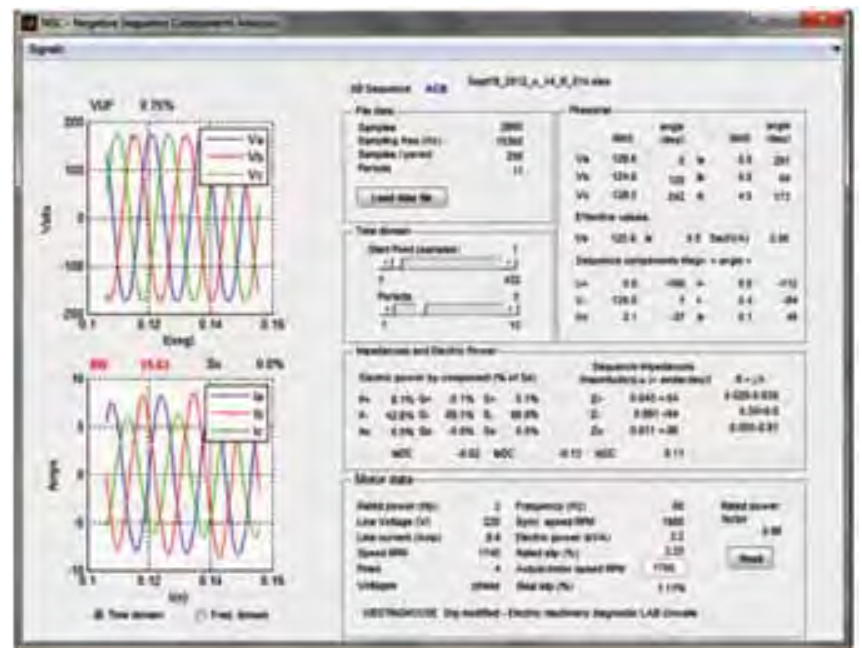

Fuente: presentación propia de los autores. 
Los resultados del primer experimento se muestran en la figura 7. En todos los casos se verificó que el factor de desequilibrio de tensiones fuera inferior al $1 \%$ para evitar la interferencia del VUF sobre la componente de asimetría constructiva. La corriente se secuencia negativa se grafica contra el número de espiras puestas en falla $(n)$.

Figura 7. Corriente de secuencia negativa para diferentes resistencias limitadoras de corriente y diferentes niveles de falla

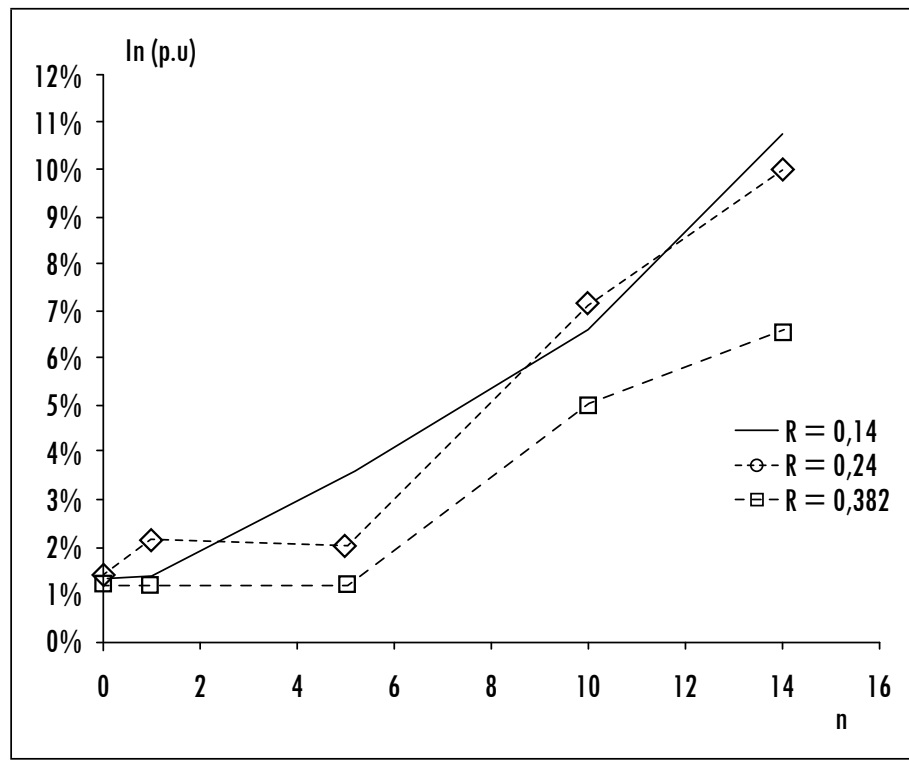

n: representa el número de espiras en corto circuito.

Fuente: presentación propia de los autores.

La corriente de secuencia negativa en p. u. se calcula como la corriente en amperios dividida entre la corriente de línea nominal del motor, la cual se toma como corriente base.

$$
I_{n-p . u}=\frac{I_{n}}{I_{\text {nom }}} \times 100 \%
$$

Se aprecia que en ausencia de falla $(n=0)$ la corriente de secuencia negativa en todos los casos tiende a ser del $1 \%$ en p. u. Este valor se identifica como el valor de corriente por asimetría inherente a la máquina bajo estudio.

Los resultados del segundo experimento se aprecian en la figura 8. En este caso se grafica la corriente de secuencia negativa en función del VUF expresado 
en porcentaje. Para obtener un desbalance de voltajes, se agrega una resistencia del orden de $0,1-0,5 \Omega$ en serie con una de las fases del estator para desequilibrar las tensiones aplicadas en el rango de 0-5\%.

Figura 8. Corriente de secuencia negativa para diferentes niveles de desequilibrio de tensión en ausencia de condiciones de falla

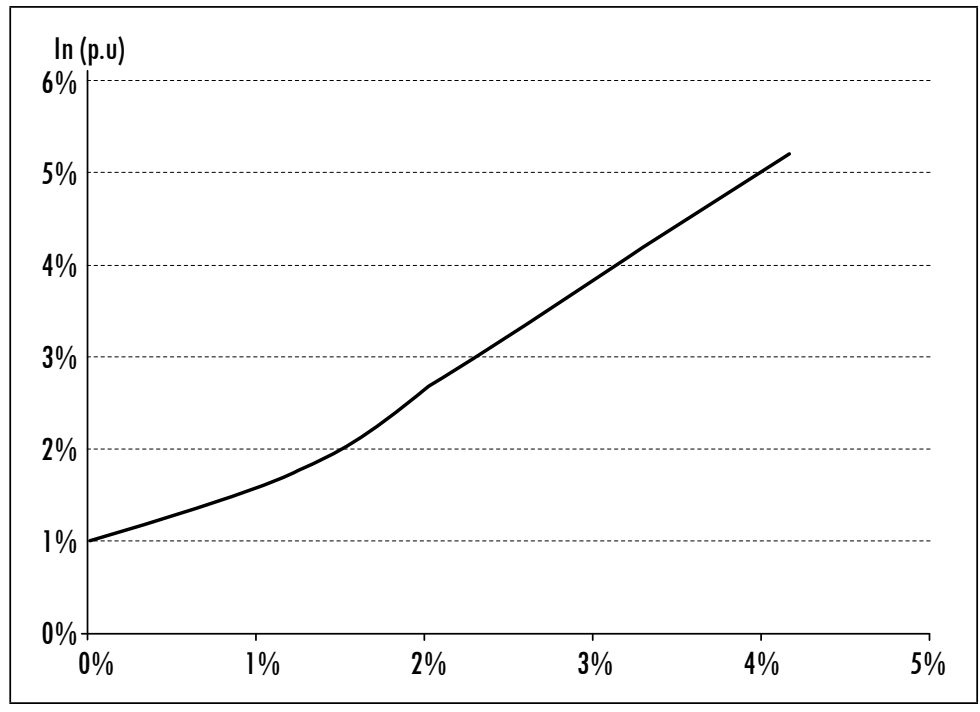

Fuente: presentación propia de los autores.

En este caso, nuevamente, se encuentra un remanente del $1 \%$ p. u. ocasionado por la asimetría inherente al motor bajo ensayo, lo cual confirma el resultado del experimento 1 . Se aprecia que existe una relación aproximadamente lineal entre el factor de desequilibrio de tensiones VUF y la corriente de secuencia negativa. Para el motor bajo ensayo, la relación encontrada fue de:

$$
I_{V U F}(p . u)=1.18 \times \operatorname{VUF}(\%)
$$

El valor reportado por Bakhri et al. (2010) para el motor analizado fue de 5,5 veces el factor de desbalance de tensiones. Esto da un margen de error grande dependiendo del motor bajo estudio.

Para el experimento final se estableció una carga igual a la nominal con ayuda de un freno dinámico basado en un generador DC y se tomaron valores de corriente de secuencia negativa, los cuales se muestran en la figura 9. Se aprecia 
cómo al incrementar el número de espiras en cortocircuito se incrementa el valor en p.u. de la corriente de secuencia negativa.

Figura 9. Corriente de secuencia negativa para diferentes niveles de falla ( $n$ ) y desequilibrio de tensión en condición de carga nominal

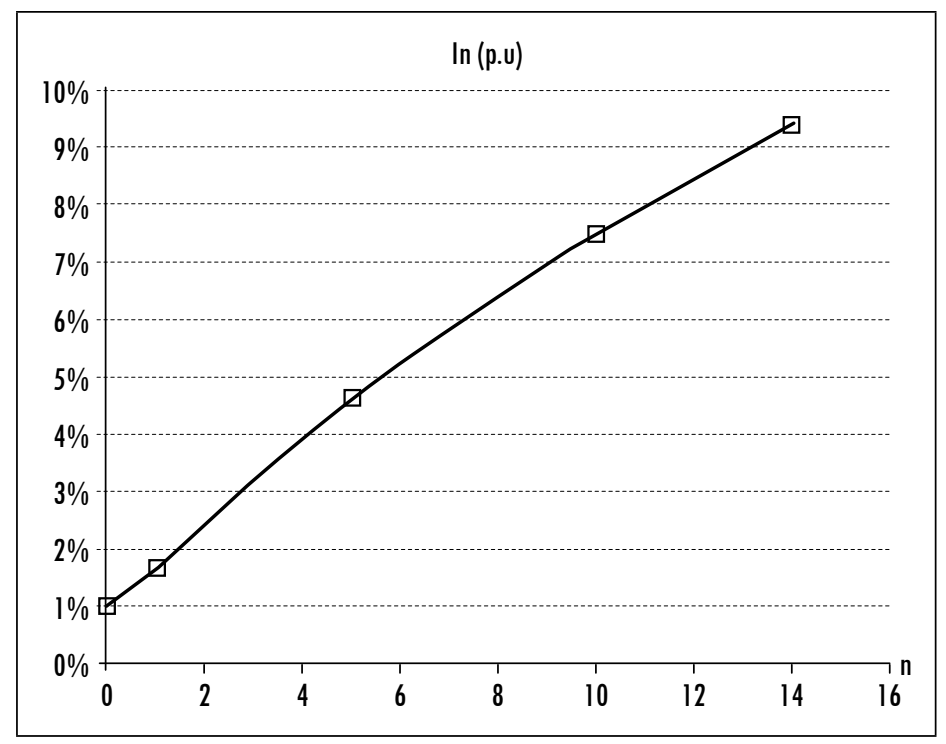

Fuente: presentación propia de los autores.

Finalmente, de la corriente de secuencia negativa obtenida en la figura 9 se descuentan las componentes de corriente inherentes a la asimetría del motor identificadas como el $1 \%$ de la corriente de secuencia negativa total, y la componente ocasionada por el desequilibrio de tensiones obtenida a partir de la figura 8, con lo que se obtiene la componente de secuencia negativa relacionada con la condición de falla, que se muestra en la figura 10.

$\mathrm{Al}$ eliminar las dos componentes y la corriente de secuencia negativa remanente presenta un valor superior al $1 \%$, se tiene un indicador de una falla inminente por cortocircuito en los devanados del motor.

Para validar la técnica se midieron varios motores en campo y se encontró evidencia de falla por cortocircuito en estator en dos motores. Se presentan los dos casos: 
Figura 10. Remanente de la corriente de secuencia negativa una vez eliminada la componente de desequilibrio de tensión y la corriente inherente a la a simetría del motor vs. nivel de falla $(n)$

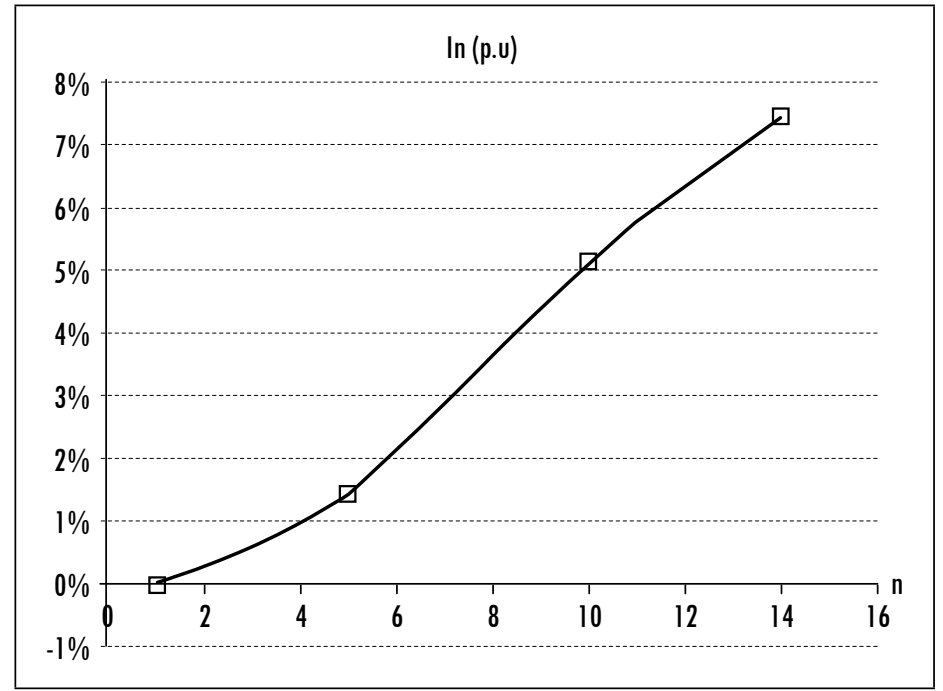

Fuente: presentación propia de los autores.

Caso 1. Un motor de $9 \mathrm{hp}, 220 \mathrm{~V}$, conexión Y sin neutro, 24 A, $3520 \mathrm{rpm}$ y $60 \mathrm{~Hz}$.

Se encontró un desbalance de tensiones del 0,22 \% y una corriente de secuencia negativa del 8,54\%. Tomando el dato más conservador de Bahkri y et al. (2010), el valor esperado para la corriente de secuencia negativa contando con asimetrías inherentes al motor y un múltiplo del desbalance de voltajes sería como máximo:

$$
\begin{aligned}
& I_{n}=I_{s f}+I_{0 n}+I_{V U F} \\
& I_{n}=I_{s f}+1 \%+5,5 * 0,22 \%=I_{s f}+2,1 \%
\end{aligned}
$$

Es decir, queda un remanente de un 6,4\% de desbalance de corriente por explicar, que debe corresponder a una falla por cortocircuito entre espiras. El motor es diagnosticado por falla en el bobinado del estator y efectivamente, al revisarlo, se encontró un daño severo en una de las bobinas de la fase A. 
Caso 2. Un motor de $150 \mathrm{hp}, 440 \mathrm{~V}$, conexión Y sin neutro, 176 A, 3780 rpm y $60 \mathrm{~Hz}$.

Se encontró un desbalance de tensiones del 0,49\% y una corriente de secuencia negativa del 6,16\%. Tomando el dato más conservador de Bahkri y et al. (2010), el valor esperado para la corriente de secuencia negativa contando con asimetrías inherentes al motor y un múltiplo del desbalance de voltajes sería como máximo:

$$
\begin{aligned}
& I_{n}=I_{s f}+I_{0 n}+I_{V U F} \\
& I_{n}=I_{s f}+1 \%+5,5 * 0,49 \%=I_{s f}+3,7 \%
\end{aligned}
$$

Es decir, queda un remanente de un 2,5\% de desbalance de corriente por explicar. En este caso, por ser relativamente pequeño el desbalance no explicado, se recomienda revisar la posibilidad de una conexión de alta resistencia en los terminales de la fase A, y en caso de descartar esa posibilidad, hacer una inspección visual del bobinado. En el momento de la publicación, la inspección visual no se había realizado.

\section{Discusión}

Se verifica que la corriente de secuencia negativa es un indicador válido de una falla incipiente en los devanados del estator de un motor de inducción; no obstante, se deben tomar en cuenta el desequilibrio de tensiones y las asimetrías inherentes a la construcción del motor. Esta observación ya había sido advertida y verificada, pero los resultados obtenidos le aportan mayor relevancia.

Un desequilibrio de tensiones puede generar la circulación de corrientes de secuencia negativa importantes, que conducen a efectos similares (en el mismo orden de magnitud) a los ocasionados por fallas de cortocircuito entre espiras, por lo que pueden dar lugar a un proceso de diagnóstico errado.

Desde el punto de vista operativo, la aplicación de los resultados obtenidos se puede hacer con base en el conocimiento que se tenga sobre los parámetros del circuito equivalente del motor; se puede reemplazar el segundo experimento por un modelo que permita predecir la componente de corriente de secuencia negativa debido al VUF y la ocasionada por asimetría inherente del motor. Estas dos componentes se pueden descontar de la corriente de secuencia negativa medida para obtener el indicador de falla.

El modelo obtenido resulta de muy bajo costo computacional, debido a la linealidad obtenida en la componente de corriente ocasionada por el desbalan- 
ce de tensiones, consistente con los hallazgos hechos por otros investigadores (Bouzid y Champenois, 2012; Bakhri et al., 2010).

\section{Conclusiones}

La corriente de secuencia negativa es un indicador fiable de las fallas en el devanado del estator por cortocircuito entre espiras, pero debe descartarse la circulación de dicha corriente, ocasionada por otros problemas como el desbalance de tensiones en la alimentación del motor.

El desbalance de tensiones, aun en pequeña escala, impulsa corrientes de secuencia negativa de magnitud considerable, comparable a la corriente de falla ocasionada por un cortocircuito entre espiras.

El método de monitoreo propuesto genera bajo costo computacional y tiene la ventaja de no ser invasivo, pero requiere un conocimiento detallado del motor para obtener un indicador fiable de falla.

\section{Referencias}

BAKHRI, S.; ERTUGRUL, N.; SOONG, W. L. y ARKAN, M. Investigation of negative sequence components for stator shorted turn detection in induction motors. Christchurch, New Zealand: The Institute of Electric and Electronics Engineers IEEE, 2010.

BOUZID, M. y CHAMPENOIS, G. Accurate stator fault detection insensitive to the unbalanced voltage in induction motor. Marseille: The Institute of Electric and Electronics Engineers, 2012.

DÍAZ, D.; DÍAZ, R.; AMAYA, M. C. y PALACIOS, J. Análisis del cortocircuito entre espiras de un motor de inducción tipo jaula de ardilla mediante la aplicación del método de elementos finitos (mef). Revista de la Facultad de IngenierÍa Universidad de Antioquia. 2010, vol. 51.

IEEE INSTRUMENTATION AND MEASUREMENT SOCIETY. IEEE guide for diagnostic field testing of electric power apparatus - electrical machinery. New York: The Institute of Electrical and Electronics Engineers, 2004.

IEEE POWER \& ENERGY SOCIETY. IEEE STD 1159-2009 Recommended practice for monitoring electric power quality. New York: The Institute of Electrical and Electronics Engineers, 2009.

IEEE POWER ENGINEERING SOCIETY. IEEE STD 112TM-Standard test procedure for polyphase induction motors and generators. New York: The Institute of Electrical and Electronics Engineers, 2004.

NANDI, S.; TOLIYAT, H. y XIAODONG, L. Condition monitoring and fault diagnosis of electrical motors—a review. New York: IEEE Transactions on Energy Conversion, 2005. 
PAZ PARRA, A et al. Consideraciones acerca del uso de la corriente de secuencia negativa como herramienta de diagnóstico de fallas estatóricas en motores de inducción de jaula de ardilla. 16 Convención Científica de Ingeniería y Arquitectura, La Habana, Cuba, 2012.

SOCIETY, ELECTRIC MACHINERY COMMITTEE OF THE IEEE POWER ENGINEERING. IEEE STD 43TM-recommended practice for testing insulation resistance of rotating machinery. New York: IEEE-SA Standards Board, 2000.

VERUCHI, C. J. y ACOSTA, G. G. Técnicas de detección y diagnostico de fallos en máquinas eléctricas de inducción. IEEE Latin America Transactions. 2007, vol. 5, pp. 41-49.

VILLADA DUQUE, F.; CADAVID, D. y VELILLA, E. Modelamiento del motor de inducción para estudios de cortocircuito entre espiras del estator. Revista Facultad de Ingeniería Universidad de Antioquia. 2010, vol. 51, pp. 7-15. 